\title{
A new approach to periodontal management
}

Amit Patel, Specialist Periodontist, tells us more about changes in the field, technologies and the importance of patient engagement.

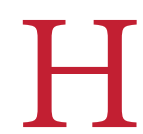

ow did periodontology and implant dentistry become areas of interest for you? I completed a training post in restorative dentistry at Liverpool Dental School after graduating as a dentist and went on to gain experience in oral and maxillofacial surgery during various hospital posts. I became a Specialist in Periodontology in 2005 in order to hone my skills and focus my practice in this area of dentistry. I have always been particularly interested in regenerative and aesthetic procedures, many of which centre around management and manipulation of the periodontal tissues.

As dental implants have grown in popularity, new challenges have become apparent in the field and I have enjoyed looking into and trying new techniques and approaches. With such a close relationship between periodontal health and implant success, it was a logical next step as I progressed my career. Teaching is another passion of mine and I find it rewarding to be able to pass on my experience in these fields and discuss ideas and concepts with like-minded colleagues.

\section{What have been some of the biggest} changes within periodontology in the last couple of years? Techniques, materials and technologies are always advancing in all areas of dentistry. It's the same in periodontology, where we have started using cutting-edge solutions to improve the treatments we provide. Today, we have state-of-the-art solutions to help reduce surgical time, improve healing and enhance aesthetic outcomes, all the while allowing for more minimally invasive techniques. The first point of call for most periodontal cases

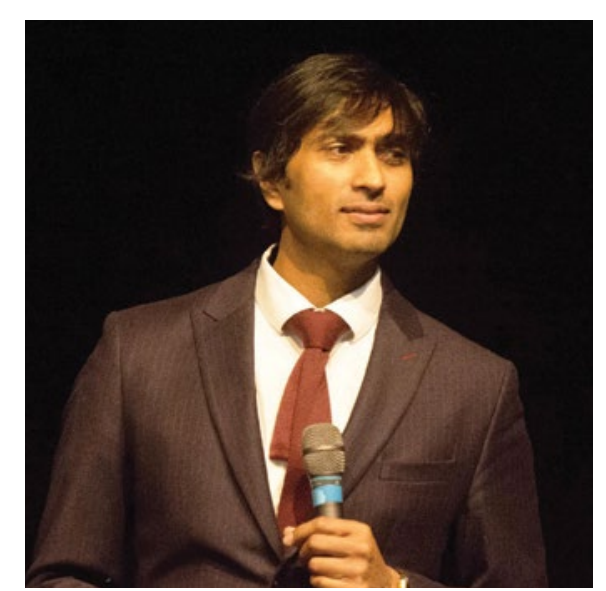

is non-surgical treatment, for which again we have a plethora of specially designed materials and instruments. The range of systems and technologies available has also made many treatments - especially dental implant therapy - more affordable and, therefore, more accessible to more patients.

However, I would say that one of the biggest single changes was the introduction of the new classification system for periodontal and peri-implant diseases and conditions. As many clinicians will be aware by now, these were the result of a World Workshop sponsored by the American Academy of Periodontology and the European Federation of Periodontology, reviewing the 1999 classification.

The previous classification was open to interpretation. Exact definitions of what constituted localised or generalised periodontitis varied slightly between practitioners. The system also didn't take into account disease progression. For example, where generalised chronic periodontitis was diagnosed, even if the disease was treated and therefore the patient's condition remained stable, the diagnosis remained the same. 
«There was no way of differentiating between stable and progressive disease.

The idea behind the new classification system was to standardise the way in which periodontal disease is diagnosed and therefore communicated between professionals. That includes between all healthcare professionals, not just those within the dental community. Once properly implemented, the new system should aid collaboration between medical, dental and maxillofacial professionals, for example, for greater consistency of patient care.

\section{'I believe one of the key aspects to successful periodontal management is patient engagement'}

What are the key points of the new classification system that clinicians need to be aware of? The new system basically follows that already used in the medical sector. As such, it offers a risk rate and a grading system. First, the condition is scored according to its severity - Stage 1 is mild and Stage 4 is very severe (Table 1). This can be calculated based on the amount of interproximal bone loss. Next, the condition is graded according to disease development - Grade A being slow and Grade C being rapid. Thanks to the recommendations set out by the BSP (British Society of Periodontology and Implant Dentistry), this is not as complex as it may initially seem. In clinical terms, the BSP suggests that a Grade A represents cases where the maximum percentage of bone loss is less than half the patient's age. When the percentage of bone loss exceeds the patient's age, they are a Grade C. Anything in between is a Grade B. The BSP has also provided a flowchart to aid diagnosis in clinical practice. ${ }^{1}$

This means that as periodontal disease develops or improves - whether on its own or in relation to dental implants - the diagnosis evolves accordingly. This enables practitioners to more accurately monitor and record active disease, tailoring treatment and management protocols over time. It also improves the communication of disease development between dental and medical professionals, where patients require multidisciplinary care. For example, this might be relevant for patients undergoing or recovering from cancer treatment.
The new periodontal classification system, like all new systems, presents some challenges for dental professionals in practice when initially introducing it. The BSP's guidelines go some way to help simplify the system and make it viable in practices, so they are certainly worth reviewing.

How else can periodontal management be improved for modern patients? I believe one of the key aspects to successful periodontal management is patient engagement. If the patient understands their current condition, what factors have contributed to it and what they can do to improve their oral health, they are more likely to comply with recommendations. It is crucial that they take responsibility for oral hygiene maintenance between dental appointments. This starts with good patient education, which is complemented by visual aids and innovative ways to encourage behaviour changes in patients.

Technology has a major role to play. I am a massive advocate of the electric toothbrush because it removes much of the user error in basic oral hygiene routines. Patients still need to know how to use it properly, but the technique is easier to achieve than with a manual toothbrush, encouraging better results. It is also important that patients use the right product - an oscillating and rotating toothbrush head is superior to its alternatives and can help patients drastically improve their periodontal condition with regular use. Many of my patients love these products and have commented on their efficacy.

Other technological innovations that encourage patient compliance should also be considered. For example, there are apps available that remind individuals to brush their teeth twice daily and help them do so effectively. They will show which areas of the mouth are focused on and indicate where time or technique may be lacking.

All of these things are about patientcentred treatment. They improve a patient's engagement with their oral health and motivate them to treat or prevent periodontal disease themselves. This, combined with more accurate monitoring and recording of disease progression, is essential for successful long-term management.

Amit will be covering these topics in greater detail within the BDA Theatre at the British Dental Conference and Dentistry Show in May. In his presentation 'Unravelling the new perio classification system', sponsored by Oral B, he will explore how to overcome the challenges faced in implementing the new classification system, while also discussing the importance of non-surgical periodontal therapy in disease management. He will also further highlight the patient's responsibility in minimising disease progression for optimal outcomes.

The British Dental Conference and Dentistry Show 2020 takes place on 15-16 May at Birmingham NEC, co-located with DTS. Register for free online. Visit www.thedentistryshow.co.uk or call 02073485270.

\section{Reference}

1. British Society of Periodontology. BSP Flowchart released. 19 January 2019. Available at: https://www. bsperio.org.uk/news/bsp-flowchart-released (accessed February 2020)

Table 1 Implementation of new periodontal classification system according to the BSP guidelines

\begin{tabular}{|c|c|c|c|c|}
\hline Staging & $\begin{array}{l}\text { Stage I } \\
\text { (Early/mild) }\end{array}$ & $\begin{array}{l}\text { Stage } 2 \\
\text { (Moderate) }\end{array}$ & $\begin{array}{l}\text { Stage } 3 \\
\text { (Severe) }\end{array}$ & $\begin{array}{l}\text { Stage } 4 \\
\text { (Very severe) }\end{array}$ \\
\hline $\begin{array}{l}\text { Maximum interproximal } \\
\text { bone loss }\end{array}$ & $<15 \%$ & Coronal third & Mid third & Apical third \\
\hline Extent & \multicolumn{4}{|c|}{$\begin{array}{l}\text { Localised - up to } 30 \% \text { of teeth } \\
\text { Generalised - more than } 30 \% \text { of teeth } \\
\text { Molar/incisor pattern }\end{array}$} \\
\hline Grading & Grade A (Slow) & $\begin{array}{l}\text { Grade B } \\
\text { (Moderate) }\end{array}$ & Grade C (Rapid) & \\
\hline $\begin{array}{l}\text { Maximum percentage bone } \\
\text { loss/patient age }\end{array}$ & $<0.5 \%$ & $0.5-1.0 \%$ & $>1.0 \%$ & \\
\hline
\end{tabular}

